AperTO - Archivio Istituzionale Open Access dell'Università di Torino

Distribution of secondary particles intensities over Earth's surface: Effect of the geomagnetic field

This is a pre print version of the following article:

Original Citation:

Availability:

This version is available http://hdl.handle.net/2318/126623

since 2016-06-30T18:54:34Z

Published version:

DOI:10.1016/j.asr.2012.06.010

Terms of use:

Open Access

Anyone can freely access the full text of works made available as "Open Access". Works made available under a Creative Commons license can be used according to the terms and conditions of said license. Use of all other works requires consent of the right holder (author or publisher) if not exempted from copyright protection by the applicable law. 


\title{
Distribution of secondary particles over Earth's surface: effect of the geomagnetic field
}

\author{
P. Bobik ${ }^{\text {a }}$, K. Kudela ${ }^{\text {a }}$, B. Pastircak ${ }^{\text {a }}$, A. Santangelo ${ }^{\text {b }}$, M. Bertaina ${ }^{\text {c }}$, K. Shinozaki ${ }^{\text {d, F. Fenu }}{ }^{\text {b }}$, J. \\ Szabelski ${ }^{\text {e }}$ J. Urbar ${ }^{\text {f }}$ \\ ${ }^{a}$ Institute of Experimental Physics SAS, Watsonova 47, 04001 Kosice, Slovak Republic \\ ${ }^{\mathrm{b}}$ Institute of Astronomy and Astrophysics, Universitt Tubingen, Sand 1, 72076 Tubingen, Germany \\ ${ }^{\text {c }}$ Department of General Physics, University of Torino, Via P. Giuria 1, 10125 Torino, Italy \\ ${ }^{\mathrm{d}}$ RIKEN Advanced Science Institute, 2-1 Hirosawa, Wako 351-0198, Japan \\ e Soltan Institute for Nuclear Studies, 90-950 Lodz, Box 447, Poland \\ ${ }^{\mathrm{f}}$ Department of Surface and Plasma Science, Charles University, V Holesovickach 2, Praha , 180 \\ 00, Czech Republic
}

\begin{abstract}
We use a Corsika package (Heck et al., 1998) and an AMS-01 flight data (Alcaraz et al., 2000) to evaluate a distribution of secondary particles in the Earth atmosphere. Distribution covers all longitudes and latitudes of STS-91 Space Shuttle flight trajectory to Mir Space Station. Moreover distribution covers all depth in the atmosphere in evaluated area. We show distributions for e-, e+, $\mu^{+}, \mu^{-}$, gammas, hadrons and Cherenkov light from primary protons component of cosmic rays flux. Our results compare favorably with other estimates made by different techniques.

We also estimate a UV light production (300-400 nm) by electron component of secondaries at the top of the atmosphere and at ISS orbit.
\end{abstract}

\section{Introduction}

Cosmic Ray (CR hereafter) particles come to the vicinity of Earth from outer space. The flux of these particles is isotropic outside Earth's magnetosphere; inside the magnetosphere the flux is affected by the strength of the geomagnetic field. The influence of the geomagnetic field is such that the flux of galactic cosmic ray at the top of the atmosphere is not constant being the highest in the polar regions and lowest in the equatorial regions. The most precise measurement of this distribution to date has been provided from an analysis of the AMS-01 data (Alcaraz et al. 2000) who published the intensities for primary and secondary protons at low orbit for ten geomagnetic regions.

The reconstruction and analysis of the AMS-01 measurements are possible by simulation using a model of external and internal geomagnetic fields (Tsyganenko96 and IGRF Tsyganenko, 1996; IGRF, 2010) together with the technique of backtracing particle trajectories in the magnetosphere (Bobik et al. 2006; Shea et al., 1965).

As the primary cosmic ray particles pass through the magnetosphere, many enter the atmosphere. These primary particles interact with nuclei of the atmospheric constituents in Earth's atmosphere and produce extensive air showers of secondary particles. Such processes can be modeled by using a Corsika package (Heck et al., 1998). Since we know the distribution of primary cosmic ray at different places at the top of the atmosphere and since we can simulate the interaction of those particles with nuclei in the atmosphere, we can evaluate the distribution of the secondary particles at different altitudes around the world.

\section{Method}

To evaluate the situation for the whole Earth we divide Earth's surface to twelve rings, every one 
with area equivalent a solid angle one steradian (see Table 1).

Table 1. Definition of centers of the 1sr rings in the geographic coordinate system.

\begin{tabular}{|l|}
\hline Table 1. \\
\hline $\begin{array}{l}\text { Center of } 1 \text { sr rings [deg] } \\
\text { (same values for north/south latitudes) }\end{array}$ \\
\hline 4.579 \\
\hline 13.859 \\
\hline 23.540 \\
\hline 34.030 \\
\hline 46.134 \\
\hline 62.731 \\
\hline
\end{tabular}

Each of those 1sr rings is divided in to 12 parts, with 30 degree longitudinal step each, to have at all 144 areas with same surface each (1/12 sr), covered all together $95.5 \%$ of Earth surface. Centers of those 144 areas we use as points for which we made our simulations (points $P_{k}$ where $\mathrm{k}=1,2, \ldots, 144$ ). At these positions we simulate showers for 31 energies $E_{i}$ equivalent to centers of AMS-01 energy bins in range from $70 \mathrm{MeV}$ to $199.06 \mathrm{GeV}$ (Alcaraz et al., 2000).

We assume incoming flux at the top of the atmosphere be angulary isotropic (see appendix 1 . for explanation of this assumption). For every of 31 energies we simulate $N$ showers with uniformly distributed incoming angles of particles with zenith angles in interval $\theta=\left(0^{\circ}, 70^{\circ}\right)$ and azimuth angles in interval $\varphi=\left(-180^{\circ}, 180^{\circ}\right)$, in acceptance cone with solid angle 4.134 sr. All together it means $144 \times 31 \times \mathrm{N}$ showers ( $N$ is equal to 800 in all simulations presented in the article). Simulations of showers were done by Corsika package version 6.960 using QGSJET II model for hadronic interactions at high energies and GHEISHA model for lower energies. Magnetic field at selected points was evaluated by IGRF model (IGRF, 2010).

Primary particle with energy $E_{i}$ create in the atmosphere at altitude $\lambda$ and position $P_{k}$ a $I_{S E C}\left(\lambda, E_{i}, x\right)$ secondary particles of type $x\left(x=e^{-}, e^{+}, \mu^{+}, \mu^{-}\right.$, hadrons and cherenkov light). Intensities $I_{S E C}\left(\lambda, E_{i}\right.$ $, x)$ are a result of Corsika simulations. For each point $P_{k}$ and energy $E_{i}$ we simulate $N$ showers for primary particles (protons) coming from isotropically distributed directions.

As primary particles we adopt particles from AMS-01 spectra. They are primary in sense that they come from magnetosphere to the top of the atmosphere. In fact, part of these particles are secondaries, produced in interactions of particles with atmosphere, escape atmosphere and because the Earth magnetic field coming back to the atmosphere (for more complex explanation see (Alcaraz et al., 2000; Aguilar et al., 2002) and downward/upward spectra therein). After

$$
I_{S E C}^{A}\left(\lambda, E_{i}, x\right)=\frac{1}{N} \sum_{j=1}^{N} I_{S E C}\left(\lambda, E_{i}, x\right)
$$

is an average intensity of secondaries from primary protons with incoming angles in interval $\theta=\left(0^{\circ}\right.$, $\left.70^{\circ}\right)$ and $\varphi=\left(-180^{\circ}, 180^{\circ}\right)$ where $N=800$. Number of primary protons $\mathrm{I}_{\text {REG }}$ in AMS region REG is taken from AMS-01 downward data. Let us note that data taken by AMS-01 detector while orbiting South Atlantic Anomaly were excluded from publication (Alcaraz et al., 2000).

Ten AMS geomagnetic regions from equatorial region 1 (geomagnetic latitude $\left|\Theta_{M}\right|<0.2$ ), region 2 (geomagnetic latitude $0.2<\left|\Theta_{\mathrm{M}}\right| \leq 0.3$ ) etc. till region 10 (geomagnetic latitude $\left|\Theta_{\mathrm{M}}\right| \geqq 1.0$ ) are taken from (Aguilar et al. 2002).

Intensity of secondary particles (type $\mathrm{x}$ ) at altitude $\lambda$ and position $P_{k}$ created by protons from i-th AMS energy bin is then 


$$
I_{i k}\left(\lambda, E_{i}, x\right)=I_{S E C}^{A}\left(\lambda, E_{i}, x\right) I_{R E G}\left(E_{i}\right) w\left(E_{i}\right)
$$

where $w\left(E_{i}\right)$ is width of i-th AMS-01 energy bin.

Because secondaries are produced by primary protons of all energies intensity of secondary particles (type x) at altitude $\lambda$ and position $P_{k}$ is

$$
I_{k}(\lambda, x)=\sum_{i=1}^{31} I_{i k}\left(\lambda, E_{i}, x\right)+I_{\text {highE }}(>199.06 \mathrm{GeV})+I_{\text {lowE }}(<0.07 \mathrm{GeV})
$$

where $I_{\text {high }}$ is the intensity of secondary particles created by primary protons with energy higher than $199.06 \mathrm{GeV}$ and $I_{\text {lowE }}$ are secondaries from primary protons with energies less than $70 \mathrm{MeV}$. Lower energies than $70 \mathrm{MeV}$ do not contribute to shower intensities in atmospheric depths higher than $20 \mathrm{~g} / \mathrm{cm}^{2}$. In equation (3) we put $I_{\text {lowE }}$ equal to zero.

Let us note that we evaluate intensities at centers of regions (points $P_{k}$ ), not for full regions area surrounded them.

The contribution of particles with energy higher than $199.06 \mathrm{GeV}$ to production of secondaries increase with increasing atmospheric depth and is different for different types of secondary particles (see Appendix II for values of $I_{\text {high }}$ and explanation). Note that $I_{\text {high }}$ contribution does not depend on geomagnetic field. For selected atmospheric depth $\lambda$ and type of secondary particles is $I_{\text {highE }}$ constant for every one of 144 selected points.

The validity of method was checked for electrons and muons by comparison with results in (Grieder, 2001) and they are qualitatively similar each other.

We made estimation for proton and helium components of the primary cosmic rays. First we use as the primary particles protons, because they make $90 \%$ of cosmic rays composition. Since published AMS downward proton intensities $I_{R E G}\left(E_{i}\right)$ are in $\left(\mathrm{m}^{2} \mathrm{~s} \mathrm{sr} \mathrm{MeV}\right)^{-1}$ and secondary particles

intensity $I_{S E C}^{A}\left(\lambda, E_{i}, x\right)$ is an averaged value for one incoming primary particle, $I_{k}(\lambda, x)$ is a number of secondary particles in shower at atmospheric depth $\lambda$ created by all primary particles from $\left(\mathrm{m}^{2} \mathrm{~s} \mathrm{sr}\right)^{-1}$ at the border of the atmosphere. But because each $\left(\mathrm{m}^{2} \mathrm{sr}\right)$ at the top of the atmosphere produces locally same value of $I_{k}(\lambda, x)$ per second, combined value of all $I_{k}(\lambda, x)$ surrounding point $\mathrm{Pk}$ gives for $\left(\mathrm{m}^{2} \mathrm{~s}\right)^{-1}$ at $\lambda$ same value as $I_{k}(\lambda, x)$. The number of secondary particles at atmospheric depth $\lambda$ is equal to a value $I_{k}(\lambda, x)$ and is in units $\left(\mathrm{m}^{2} \mathrm{~s}\right)^{-1}$. To estimate a number of UV photons produced by electron component of showers in range 300-400 $\mathrm{nm}$ we use an International Standard Atmosphere model (see web cited page) and estimation from fluorescence yield measurements by AIRFLY experiment (Arciprete et al., 2005). With setting a fluorescence yield to value 3 we estimate an upper limit of UV production by electron shower

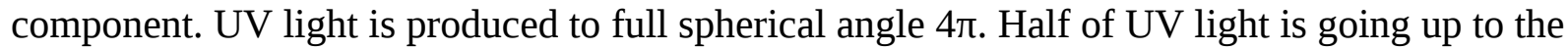
space, half to the Earth surface. Reflection of UV light depends on albedo of Earth surface which varies between few percent (conifer forest during summer has albedo 0.08 ) to almost $100 \%$ for some surfaces (fresh snow has albedo 0.8-0.9). Because UV light produced by secondary electrons is produced everywhere in the atmosphere, the intensity of UV light at different altitudes over atmosphere is a simple function of $r^{2} / r_{E}^{2}$, where $r$ is the distance from the Earth center and $r_{E}$ is the Earth radius.

\section{Results}

To illustrate effect of geomagnetic field to secondary particles production in atmosphere we show at figure 1. a dependency of electrons intensity at different depths of atmosphere for four selected points with same longitude $15^{\circ}$ and latitudes $4.58^{\circ}, 34.03^{\circ}, 46.13^{\circ}, 62.73^{\circ}$. Due to distribution of primaries with more particles at higher latitude, we see also higher number of secondaries at higher latitudes. 


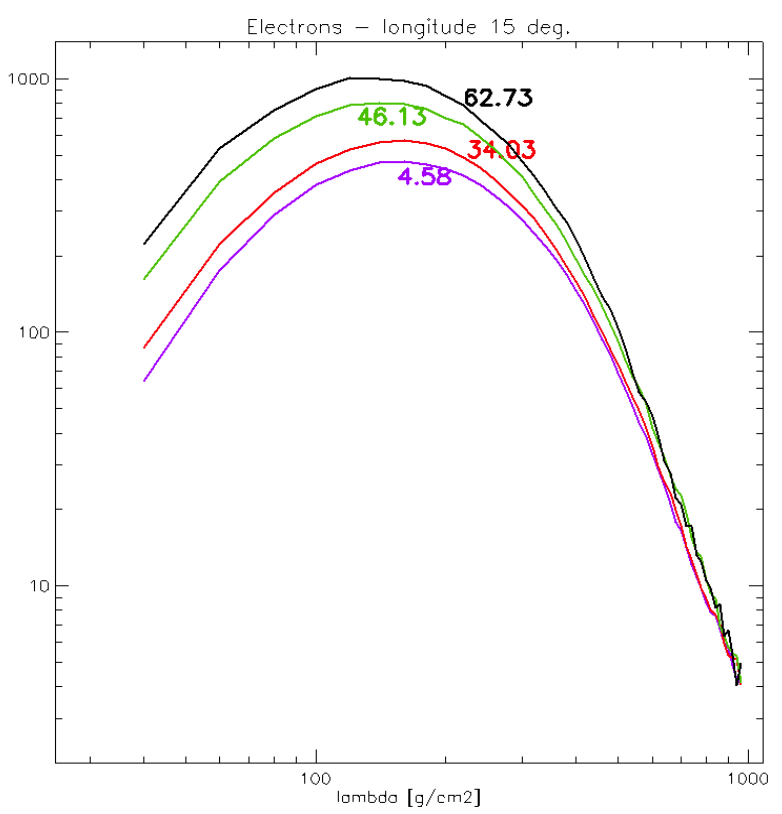

Figure 1. Dependency of secondary electrons intensity $\left(\right.$ in $\left.\left(\mathrm{m}^{2} \mathrm{~s}\right)^{-1}\right)$ produced by primary protons at atmospheric depth for selected points on Earth with geographic longitude $15^{\circ}$ and latitudes $4.58^{\circ}$, $34.03^{\circ}, 46.13^{\circ}, 62.73^{\circ}$

On figure 2. we present a distribution of Cherenkov light created by proton component of the primary CR in range 300-450nm at atmospheric depth $\lambda$ equal $200 \mathrm{~g} / \mathrm{cm}^{2}$ (left panel) and 1000 $\mathrm{g} / \mathrm{cm}^{2}$ (right panel). The Cherenkov light intensity varies with different latitudes and longitudes (see figure 2.). This variation is in two orders of magnitude smaller than same variation of primary cosmic rays intensity on the top of atmosphere. While intensity of primary protons over the atmosphere varies between region 1 and region 10 of AMS data more than 250 times, Cherenkov light intensity increasing just $\sim 70 \%$ in region 10 from level in region 1 at $1000 \mathrm{~g} / \mathrm{cm}^{2}$. The reason is that highest number of the secondary particles is created by primary particles with energies higher than cutoff rigidity of region 1 . While number of secondary particles created by one primary proton increase with increasing proton energy, number of protons in CR spectrum rapidly fall for energies over $10 \mathrm{GV}$ what is energy close to cutoff rigidity in region 1 . Thus production curve of secondaries has maximum for energies few $\mathrm{GeV}$ higher than cutoff rigidity of region with maximum geomagnetic shielding. 

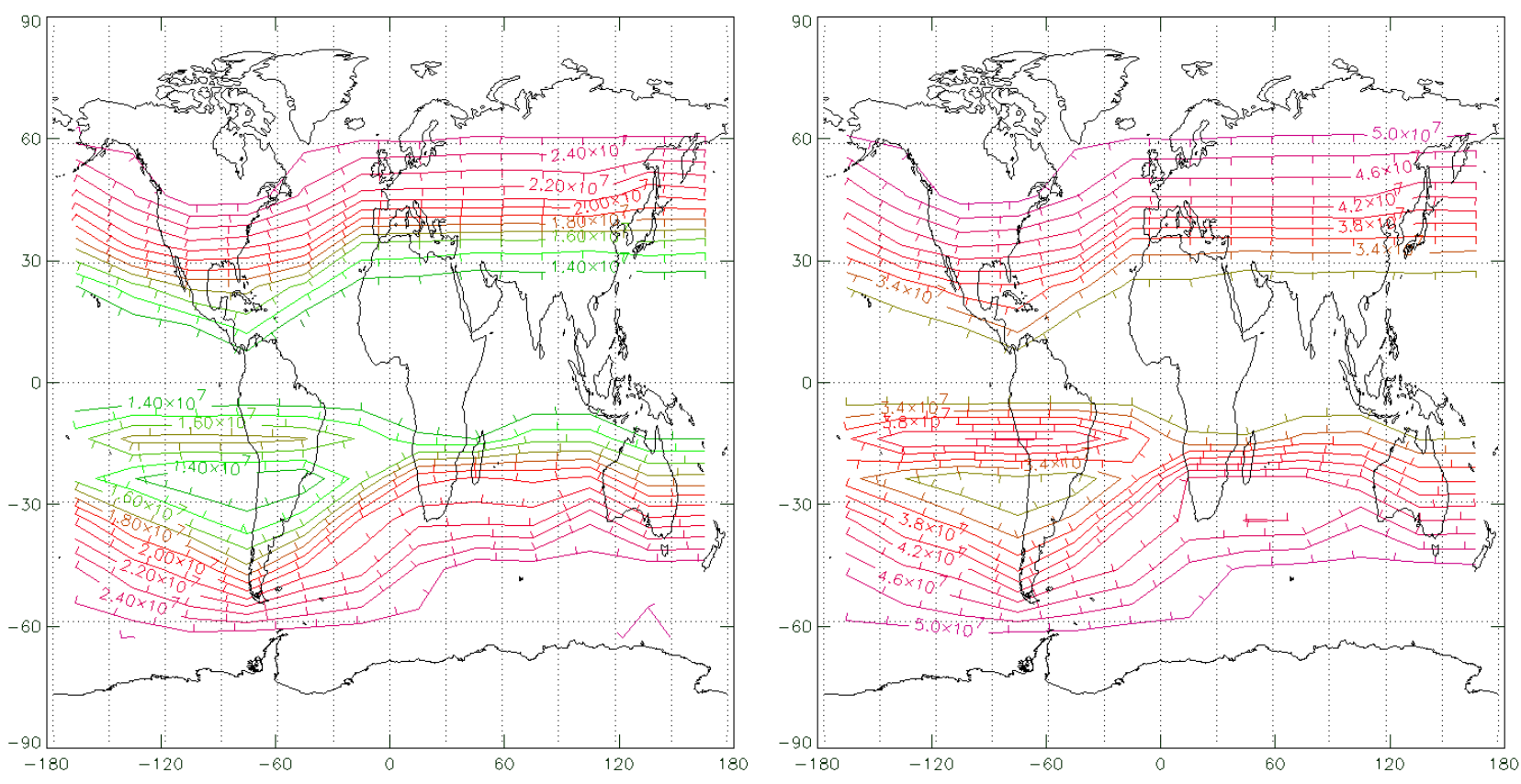

Figure 2. Cherenkov light distribution in $\left(\mathrm{m}^{2} \mathrm{~s}\right)^{-1}$ produced by primary protons at atmospheric depth $\lambda$ equal to $200 \mathrm{~g} / \mathrm{cm}^{2}$ (top panel) and $1000 \mathrm{~g} / \mathrm{cm}^{2}$ (bottom panel). Maps are in geographic coordinates.

At figure 3. we show distribution of secondary electrons created by proton component of primary CR at atmospheric depth $\lambda$ equal to $200 \mathrm{~g} / \mathrm{cm}^{2}$ (left panel) and $1000 \mathrm{~g} / \mathrm{cm}^{2}$ (right panel). Variation of intensity is similar as one for Cherenkov light at level of $200 \mathrm{~g} / \mathrm{cm}^{2}$. At $1000 \mathrm{~g} / \mathrm{cm}^{2}$ level the picture is more chaotic due to low statistic of Corsika simulation at this atmospheric depth. For more smooth picture at this $\lambda$ we need simulation with higher number of showers (higher than 800 presented here) in each of 144 selected points.
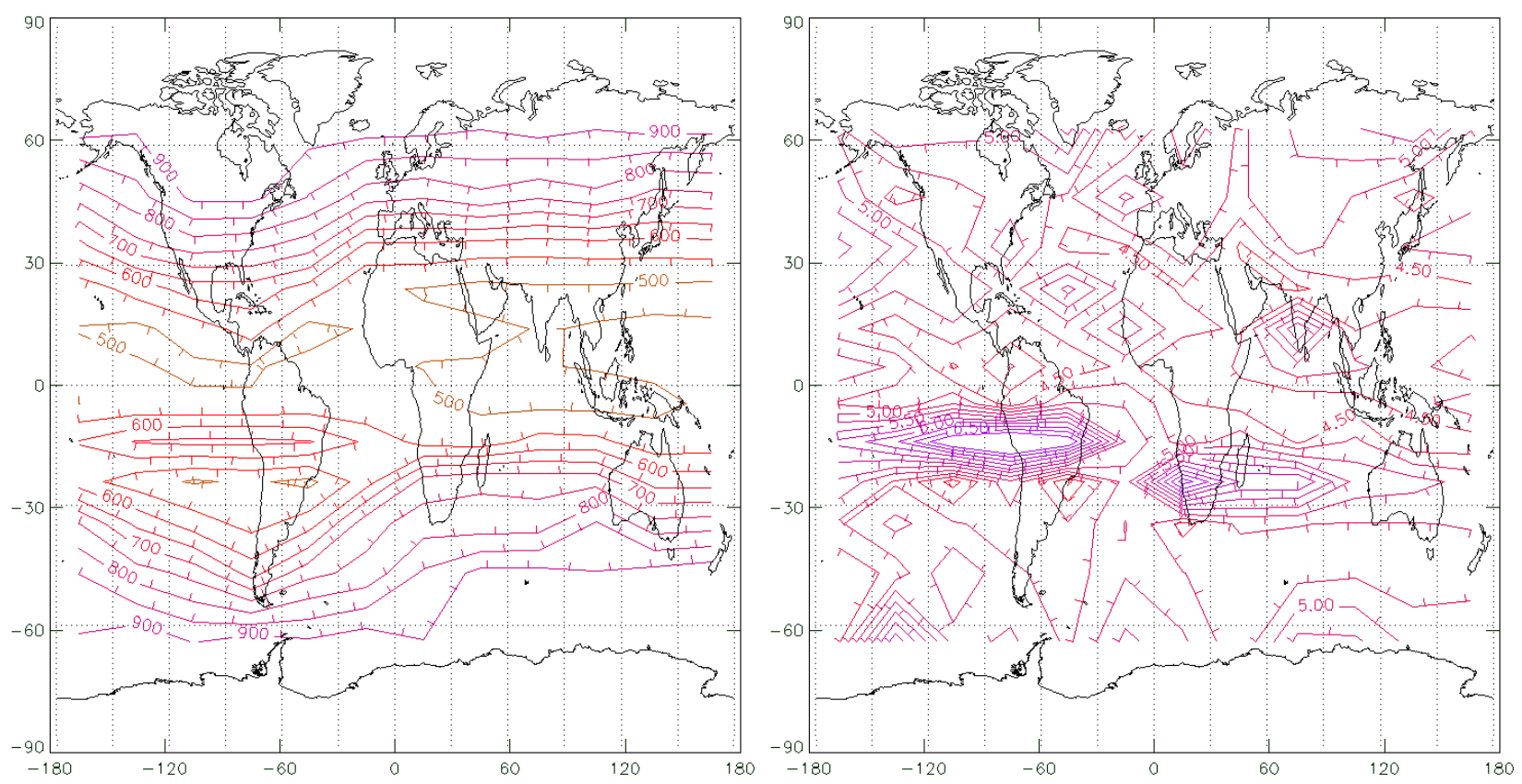

Figure 3. Secondary electrons distribution in $\left(\mathrm{m}^{2} \mathrm{~s}\right)^{-1}$ produced by primary protons at atmospheric depth $\lambda$ equal to $200 \mathrm{~g} / \mathrm{cm}^{2}$ (top panel) and $1000 \mathrm{~g} / \mathrm{cm}^{2}$ (bottom panel). Maps are in geographic coordinates. 
Gamma rays produced in the atmosphere by proton component of the primary CR we present at figure 4 and $\mu^{+}$mesons at figure 5 .
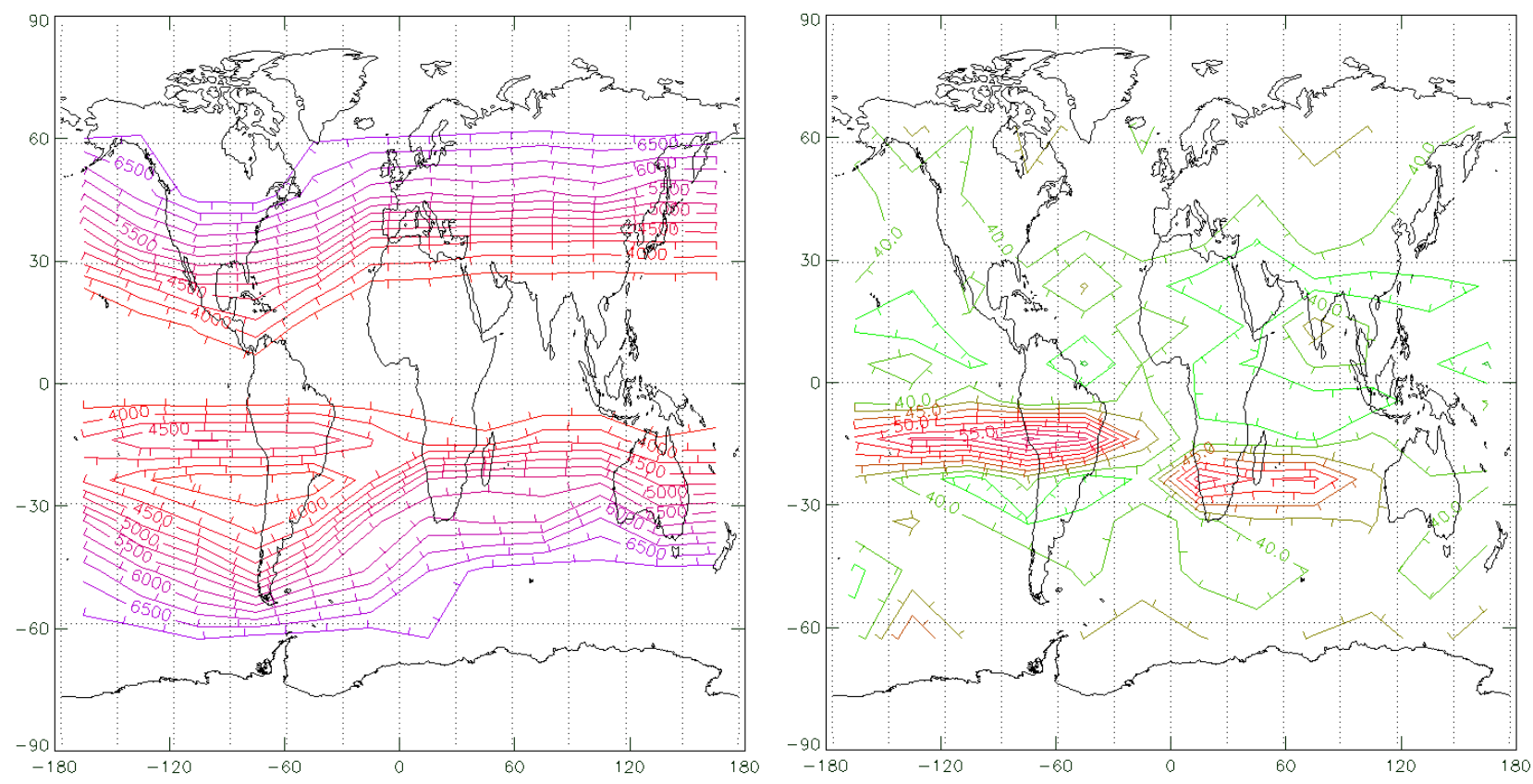

Figure 4. Gamma rays distribution in $\left(\mathrm{m}^{2} \mathrm{~s}\right)^{-1}$ at atmospherical depth $\lambda$ equal to $200 \mathrm{~g} / \mathrm{cm}^{2}$ (left panel) and $1000 \mathrm{~g} / \mathrm{cm}^{2}$ (right panel). Maps are in geographic coordinates.
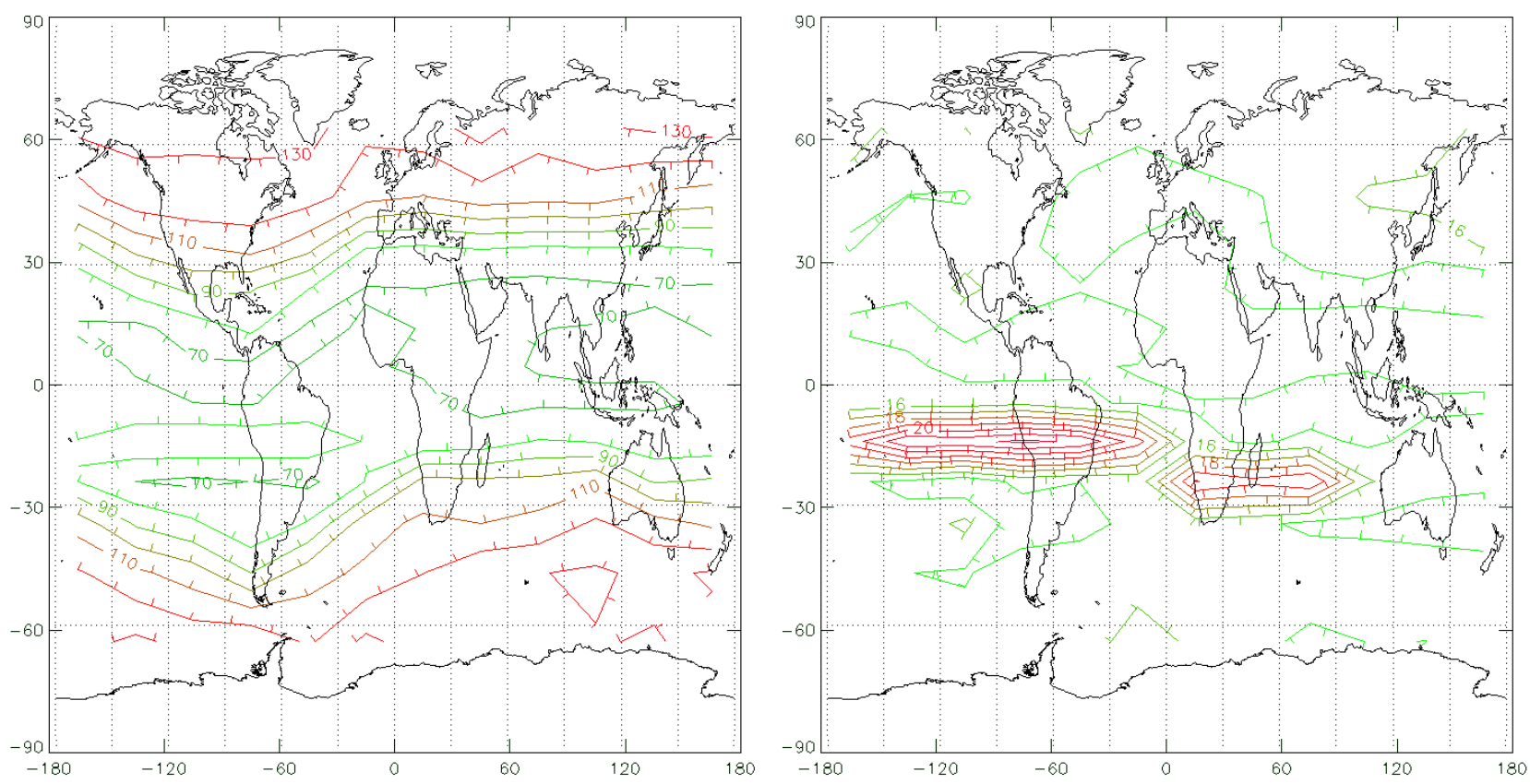

Figure 5. muons $\mathrm{s}^{+}$distribution in $\left(\mathrm{m}^{2} \mathrm{~s}\right)^{-1}$ at atmospherical depth $\lambda$ equal to $200 \mathrm{~g} / \mathrm{cm}^{2}$ (top panel) and $1000 \mathrm{~g} / \mathrm{cm}^{2}$ (bottom panel). Maps are in geographic coordinates.

UV light at the top of the atmosphere for albedo 1.0 (reflection 100\%) is presented at figure 6 . Albedo 1.0 is highly unrealistic value, what we use to set upper limit for UV light production by electrons produced by cosmic rays in the atmosphere. UV light at low orbit (400km altitude) will have the value 0.885 times level at the top of the atmosphere. Intensities of UV light created by cosmic rays are relatively very small to other sources (nightglow, zodiacal light, integrated star 
light) of UV light also in cases when we speak about night side of Earth atmosphere.

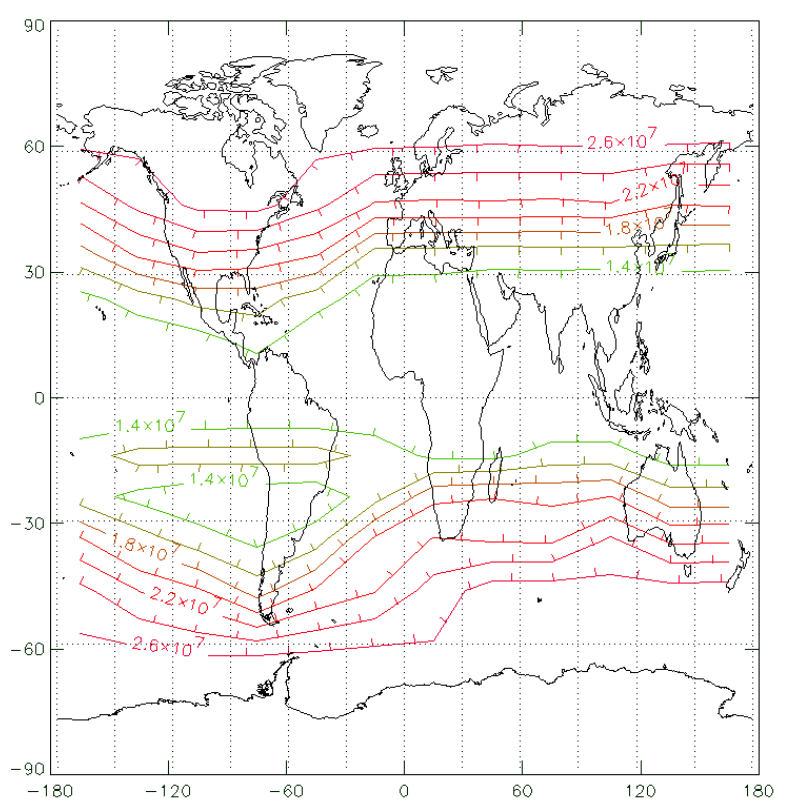

Figure 6. UV at the top of the atmosphere for albedo 100\%. For albedo $0 \%$ UV light in 300-400nm (number of photons in $\left(\mathrm{m}^{2} \mathrm{~s}\right)^{-1}$ ) will have half values. Map is in geographic coordinates.

Table 2. show places where minimum and maximum level of secondaries production (from proton component of the primary CR) in the atmosphere appear for selected levels of atmospheric depth. Places here mean centers of regions on Earth surface with area 1/12 of steradian of full angle $4 \pi$ from all Earth surface. Intensities presented in table are overall intensities (flux for all energies), for Cherenkov light it is in the range of $300-450 \mathrm{~nm}$.

Table 2. Minima and maxima of secondary particles intensity at atmospheric depths 200, 700 and $1000 \mathrm{~g} / \mathrm{cm}^{2}$ created by proton component of primary CR.

\begin{tabular}{|c|c|c|c|c|c|c|c|}
\hline & \multicolumn{3}{|c|}{ Minimum } & \multicolumn{3}{|c|}{ Maximum } & \multirow[b]{2}{*}{$\begin{array}{l}\text { Max./Min } \\
\text { ratio }\end{array}$} \\
\hline & $\begin{array}{l}\text { latitude } \\
\text { deg. }\end{array}$ & $\begin{array}{l}\text { longitude } \\
\text { deg. }\end{array}$ & $\begin{array}{l}\text { intensity } \\
\left(\mathrm{m}^{2} \mathrm{~s}\right)^{-1}\end{array}$ & $\begin{array}{l}\text { latitude } \\
\text { deg. }\end{array}$ & $\begin{array}{l}\text { longitude } \\
\text { deg. }\end{array}$ & $\begin{array}{l}\text { intensity } \\
\left(\mathrm{m}^{2} \mathrm{~s}\right)^{-1}\end{array}$ & \\
\hline Cherenkov light - $1000 \mathrm{~g} / \mathrm{cm}^{2}$ & -4.58 & 105.00 & $3.03 \times 10^{7}$ & -62.73 & 135.00 & $5.15 \times 10^{7}$ & 1.698 \\
\hline Cherenkov light - $200 \mathrm{~g} / \mathrm{cm}^{2}$ & 13.86 & -45.00 & $1.30 \times 10^{7}$ & -62.73 & 135.00 & $2.63 \times 10^{7}$ & 2.020 \\
\hline electrons - $1000 \mathrm{~g} / \mathrm{cm}^{2}$ & -23.54 & -45.00 & 4.01 & -13.86 & -75.00 & 7.52 & 1.875 \\
\hline electrons - $200 \mathrm{~g} / \mathrm{cm}^{2}$ & 13.86 & -45.00 & $4.82 \times 10^{2}$ & -46.13 & 165.00 & $9.40 \times 10^{2}$ & 1.950 \\
\hline gamma - $1000 \mathrm{~g} / \mathrm{cm}^{2}$ & 4.58 & -45.00 & $3.45 \times 10^{1}$ & -13.86 & -75.00 & $6.04 \times 10^{1}$ & 1.751 \\
\hline gamma - $200 \mathrm{~g} / \mathrm{cm}^{2}$ & 13.86 & -45.00 & $3.51 \times 10^{3}$ & -62.73 & 135.00 & $6.98 \times 10^{3}$ & 1.987 \\
\hline muon $^{+}-1000 \mathrm{~g} / \mathrm{cm}^{2}$ & -4.58 & 105.00 & $1.41 \times 10^{1}$ & -13.86 & -75.00 & $2.22 \times 10^{1}$ & 1.574 \\
\hline muon $^{+}-200 \mathrm{~g} / \mathrm{cm}^{2}$ & 23.54 & 75.00 & $6.59 \times 10^{1}$ & 62.73 & 45.00 & $1.34 \times 10^{2}$ & 2.026 \\
\hline muon $^{-}-1000 \mathrm{~g} / \mathrm{cm}^{2}$ & 46.13 & 75.00 & $1.09 \times 10^{1}$ & -13.86 & -135.00 & $1.84 \times 10^{1}$ & 1.688 \\
\hline muon $^{-}-200 \mathrm{~g} / \mathrm{cm}^{2}$ & 23.54 & 75.00 & $5.47 \times 10^{1}$ & -62.73 & 105.00 & $9.92 \times 10^{1}$ & 1.814 \\
\hline positrons $-1000 \mathrm{~g} / \mathrm{cm}^{2}$ & -23.54 & -45.00 & 2.58 & -13.86 & -75.00 & 4.89 & 1.895 \\
\hline positrons - $200 \mathrm{~g} / \mathrm{cm}^{2}$ & -23.54 & -45.00 & $3.32 \times 10^{2}$ & -62.73 & -15.00 & $6.38 \times 10^{2}$ & 1.922 \\
\hline hadrons $-1000 \mathrm{~g} / \mathrm{cm}^{2}$ & 23.54 & 75.00 & $9.00 \times 10^{-1}$ & 62.73 & 105.00 & 3.43 & 3.811 \\
\hline hadrons - $200 \mathrm{~g} / \mathrm{cm}^{2}$ & 23.54 & 75.00 & $1.52 \times 10^{2}$ & 62.73 & -45.00 & $1.00 \times 10^{3}$ & 6.579 \\
\hline Cherenkov light - $700 \mathrm{~g} / \mathrm{cm}^{2}$ & -4.58 & 75.00 & $2.91 \times 10^{7}$ & -62.73 & 135.00 & $5.03 \times 10^{7}$ & 1.729 \\
\hline electrons - $700 \mathrm{~g} / \mathrm{cm}^{2}$ & -4.58 & 105.00 & $2.08 \times 10^{1}$ & -13.86 & -75.00 & $3.25 \times 10^{1}$ & 1.563 \\
\hline
\end{tabular}




\begin{tabular}{|l|l|l|l|l|l|l|l|}
\hline${\text { gamma }-700 \mathrm{~g} / \mathrm{cm}^{2}}$ & -4.58 & 135.00 & $1.97 \times 10^{2}$ & -13.86 & -75.00 & $2.97 \times 10^{2}$ & 1.508 \\
\hline muon $^{+}-700 \mathrm{~g} / \mathrm{cm}^{2}$ & -23.54 & -105.00 & $2.32 \times 10^{1}$ & -13.86 & -75.00 & $3.51 \times 10^{1}$ & 1.513 \\
\hline muon $^{-}-700 \mathrm{~g} / \mathrm{cm}^{2}$ & -4.58 & 75.00 & $1.84 \times 10^{1}$ & -13.86 & -135.00 & $2.98 \times 10^{1}$ & 1.620 \\
\hline positrons $-700 \mathrm{~g} / \mathrm{cm}^{2}$ & 4.58 & 165.00 & $1.33 \times 10^{1}$ & -13.86 & -75.00 & $2.12 \times 10^{1}$ & 1.594 \\
\hline hadrons $-700 \mathrm{~g} / \mathrm{cm}^{2}$ & 23.54 & 75.00 & 8.66 & 62.73 & 105.00 & $3.00 \times 10^{1}$ & 3.464 \\
\hline
\end{tabular}

To estimate an influence of heavier components of the primary CR to production of secondary CR in the Earth atmosphere we evaluate the same set of simulation also for helium. We use AMS-01 helium spectra (Aguilar et al., 2002) as primary helium spectra for three geomagnetic superregions (Bobik et al., 2009). The results show that intensity of produced secondary particles from primary helium is more important in the upper layers of atmosphere. The ratio of electron component of secondary CR created by primary helium to electrons created by primary protons is between 2 and $4 \%$ at atmospheric depth $1000 \mathrm{~g} / \mathrm{cm}^{2}$, depending on place on the Earth. Very similar results we get for muons. For hadron component this ratio of secondary CR is from 4 to $10 \%$ at $1000 \mathrm{~g} / \mathrm{cm}^{2}$. The ratio rise with increasing altitude in atmosphere. Particularly, at atmospheric depth $200 \mathrm{~g} / \mathrm{cm}^{2}$ electrons from primary protons to primary helium ratio rise to the range from 6 till 10\%. The situation for muons is similar. In hadrons the ratio varies from 8 to $27 \%$.

Table 3. show intensities of secondary CR from helium primary CR maxima and minima at Earth surface.

Table 3. Minima and maxima of secondary particles intensity at atmospheric depths 200, 700 and $1000 \mathrm{~g} / \mathrm{cm}^{2}$ created by helium component of primary CR.

\begin{tabular}{|c|c|c|c|c|c|c|c|}
\hline & \multicolumn{3}{|c|}{ Minimum } & \multicolumn{3}{|c|}{ Maximum } & \multirow[b]{2}{*}{$\begin{array}{l}\text { Max./Min } \\
\text { ratio }\end{array}$} \\
\hline & $\begin{array}{l}\text { latitude } \\
\text { deg. }\end{array}$ & $\begin{array}{l}\text { longitude } \\
\text { deg. }\end{array}$ & $\begin{array}{l}\text { intensity } \\
\left(\mathrm{m}^{2} \mathrm{~s}\right)^{-1}\end{array}$ & $\begin{array}{l}\text { latitude } \\
\text { deg. }\end{array}$ & $\begin{array}{l}\text { longitude } \\
\text { deg. }\end{array}$ & \begin{tabular}{|l|} 
intensity \\
$\left(\mathrm{m}^{2} \mathrm{~s}\right)^{-1}$
\end{tabular} & \\
\hline Cherenkov light - $1000 \mathrm{~g} / \mathrm{cm}^{2}$ & 4.58 & -135 & $3.87 \times 10^{3}$ & $-46,13$ & 45 & $4.22 \times 10^{3}$ & 1.090 \\
\hline Cherenkov light - $200 \mathrm{~g} / \mathrm{cm}^{2}$ & 4.58 & -105 & $1.48 \times 10^{5}$ & 62,73 & -135 & $1.90 \times 10^{5}$ & 1.286 \\
\hline electrons - $1000 \mathrm{~g} / \mathrm{cm}^{2}$ & 4.58 & -135 & $2.85 \times 10^{-1}$ & 46,13 & -15 & $3.41 \times 10^{-1}$ & 1.196 \\
\hline electrons - $200 \mathrm{~g} / \mathrm{cm}^{2}$ & 4.58 & -105 & $4.19 \times 10^{1}$ & $-62,73$ & 105 & $5.70 \times 10^{1}$ & 1.360 \\
\hline gamma - $1000 \mathrm{~g} / \mathrm{cm}^{2}$ & 4.58 & -135 & 2.34 & $-23,54$ & 75 & 2.71 & 1.158 \\
\hline gamma - $200 \mathrm{~g} / \mathrm{cm}^{2}$ & 4.58 & -105 & $1.02 \times 10^{3}$ & $-62,73$ & -45 & $1.14 \times 10^{3}$ & 1.118 \\
\hline muon $^{+}-1000 \mathrm{~g} / \mathrm{cm}^{2}$ & 4.58 & -165 & $8.58 \times 10^{-1}$ & 34,03 & -165 & $9.64 \times 10^{-1}$ & 1,124 \\
\hline muon $^{+}-200 \mathrm{~g} / \mathrm{cm}^{2}$ & 4.58 & -165 & 5.32 & $-34,03$ & -135 & 6.44 & 1.211 \\
\hline muon $^{-}-1000 \mathrm{~g} / \mathrm{cm}^{2}$ & 4.58 & -135 & $8.43 \times 10^{-1}$ & 34,03 & -135 & $9.42 \times 10^{-1}$ & 1.117 \\
\hline muon $^{-}-200 \mathrm{~g} / \mathrm{cm}^{2}$ & 4.58 & -135 & 5.50 & $-13,86$ & 75 & 6.82 & 1.240 \\
\hline positrons - $1000 \mathrm{~g} / \mathrm{cm}^{2}$ & 4.58 & -135 & $1.79 \times 10^{-1}$ & $-62,73$ & 45 & $2.17 \times 10^{-1}$ & 1.212 \\
\hline positrons $-200 \mathrm{~g} / \mathrm{cm}^{2}$ & 4.58 & -105 & $2.85 \times 10^{1}$ & $-62,73$ & 105 & $3.87 \times 10^{1}$ & 1.358 \\
\hline hadrons $-1000 \mathrm{~g} / \mathrm{cm}^{2}$ & 13.86 & 105 & $4.24 \times 10^{-2}$ & 34,03 & 165 & $7.41 \times 10^{-2}$ & 1.748 \\
\hline hadrons - $200 \mathrm{~g} / \mathrm{cm}^{2}$ & 4.58 & -105 & $2.95 \times 10^{1}$ & $-46,13$ & 15 & $6.14 \times 10^{1}$ & 2.081 \\
\hline
\end{tabular}

\section{Conclusion}

We present distribution of secondary particles in Earth atmosphere created by primary cosmic rays based on AMS-01 published data and Corsika package simulation results. We evaluate a variation 
of secondary particles intensity with location at Earth in AMS-01 geomagnetic regions. Intensity of secondary particles varies from low geomagnetic regions to regions with higher geomagnetic latitudes in order a hundreds percents, what is reasonably less than variation of primary cosmic rays at the border of the atmosphere.

Because of characteristic production of secondary particles, dependency on solar cycle should be smaller than for primary particles.

\section{Acknowledgments}

This work was supported by Slovak Academy of Sciences MVTS JEM-EUSO.

\section{Appendix 1.}

We test validity of isotropic angles distribution assumption for incoming particles at the top of the atmosphere. Validity in sense of influence to provided simulation. To estimate importance of different angle distribution of incoming primary particles to production of secondary particles we made calculation for particles coming from different sectors of cone with $\theta=\left(0^{\circ}, 70^{\circ}\right)$ and $\varphi=(-$ $180^{\circ}, 180^{\circ}$ ). Incoming angles in different sectors are isotropically distributed. Sectors with ranges of $\varphi=\left(0^{\circ}, 180^{\circ}\right),\left(180^{\circ}, 0^{\circ}\right),\left(-90^{\circ}, 90^{\circ}\right),\left(90^{\circ},-90^{\circ}\right)$. Results for all sectors are very similar to those for $\varphi$ $=\left(-180^{\circ}, 180^{\circ}\right)$. Difference in resulted intensities are no more than a few percent.

The AMS-01 published data was combined from data collected during three periods corresponding to shuttle attitude $0^{\circ}, 20^{\circ}$ and $45^{\circ}$ because measurements show agreement with an isotropic distribution (see Fig. 4.3. (a) in (Aguilar et al., 2002)). This allows us use isotropic distribution in angular range of AMS-01 measurements. To see difference between maximum allowed zenith angle in Corsika $\theta=70^{\circ}$ and AMS $\theta=45^{\circ}$ we evaluate secondary particle flux dependence on altitude $\lambda$ at two points on Earth, one in region 1, second in region 10. Difference in order of tenths percent show importance use a maximum allowed Corsika zenith angle in simulations.

Alternative approach (Masarik and Beer, 1999) is a simulation based on cut-off evaluation (Störmer equation (Störmer, 1930) and GCR spectrum where trajectories under cutoff are excluded from simulation. Such approach uses a more precise angle distribution for incoming primary particles, but does not allow using an AMS data. This approach uses simplifications in cut-off evaluation and in primary particle spectrum evaluation.

By method presented in (Bobik et al., 2006) is possible to evaluate very precise spectra of incoming primary particles with dependency on zenith and azimuth angles and use them as input for Corsika simulation. This approach depends on simulation of primary particles spectra which we will be present in another future article.

\section{Appendix 2.}

The example of dependency $I_{i k}\left(\lambda, E_{i}, x\right)$ for electrons on $E_{i}$ presented at figure 7 . for two selected points (first from region 1 second from region 10) show that for high energies

$I_{i k}\left(\lambda, E_{i}, x\right)$ does not depend on the position on Earth. This shows that for selected $\lambda$ remains $I_{\text {high }}$ constant. Then, it is enough to evaluated $I_{h i g h E}$ in one selected point and use it for all other points. Values of $I_{\text {highe }}$ are presented in table 4. 


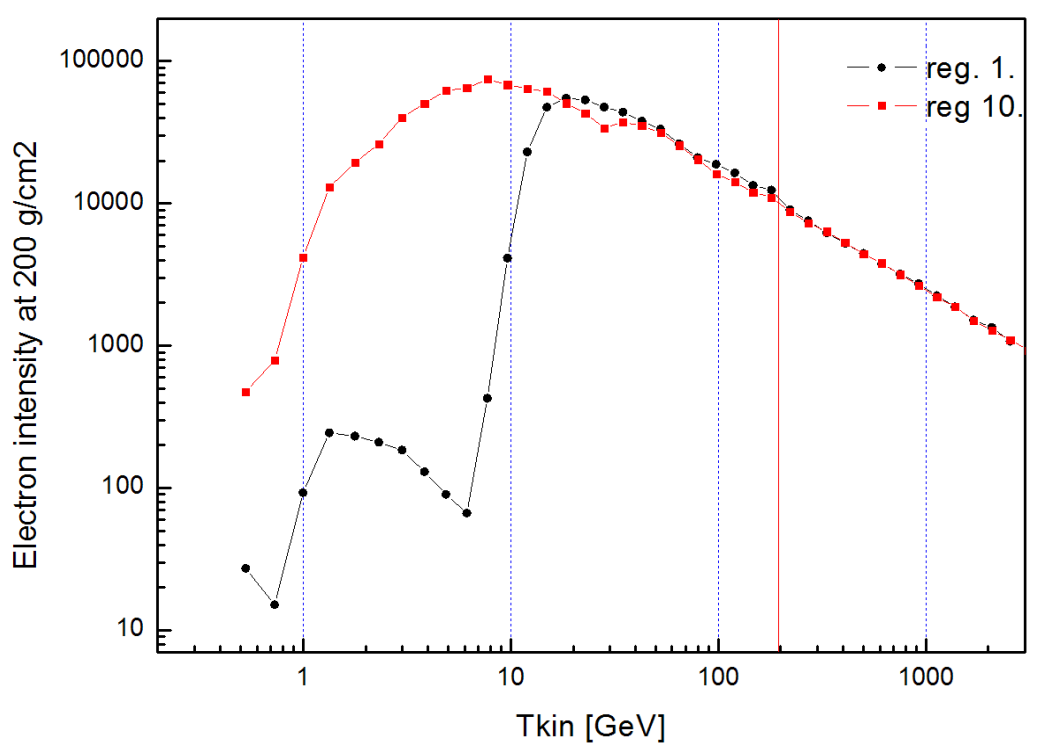

Figure 7. $I_{i k}\left(\lambda, E_{i}, x\right)$ dependency for electrons on kinetic energy Ei for selected point from region 1 and region 10 at atmospheric depth $\lambda$ equal to $200 \mathrm{~g} / \mathrm{cm}^{2}$.

Table 4. Intensity of secondary particles at atmospheric depth 200 and $1000 \mathrm{~g} / \mathrm{cm}^{2}$ created by primary protons with energy higher than $199.06 \mathrm{GeV}$.

\begin{tabular}{|l|l|l|}
\hline \multirow{2}{*}{} & $I_{\text {highe }}$ in $\left[\left(\mathrm{m}^{2} \mathrm{~s}\right)^{-1}\right]$ \\
\cline { 2 - 3 } & at $\lambda=200 \mathrm{~g} / \mathrm{cm}^{2}$ & at $\lambda=1000 \mathrm{~g} / \mathrm{cm}^{2}$ \\
\hline Cherenkov light & $1.26 \times 10^{6}$ & $5.05 \times 10^{6}$ \\
\hline Gamma & $3.76 \times 10^{2}$ & 9.64 \\
\hline electrons & $5.70 \times 10^{1}$ & 1.17 \\
\hline hadrons & 3.15 & $8.71 \times 10^{-2}$ \\
\hline muons+ & 4.51 & 2.49 \\
\hline muons- & 4.39 & 2.50 \\
\hline positrons & $4.00 \times 10^{1}$ & $7.18 \times 10^{-1}$ \\
\hline
\end{tabular}

The figure 7. shows also that maximum of $I_{i k}\left(\lambda, E_{i}, x\right)$ for electrons for region 10 is close to $10 \mathrm{GeV}$. For another region maximum will have higher value. Because over $10 \mathrm{GeV}$ is spectrum of primary protons almost not modulated by heliosphere, we can expect small variation of secondaries electron component during solar cycle in comparison with variation of primary particles intensity during solar cycle. This modulation depends on number of secondary particles created by part of primary spectrum under $10 \mathrm{GeV}$. Evaluation of number of secondaries created by primary particles with energy lower than $10.73 \mathrm{GeV}$ (upper border of 17th AMS-01 energy bin) shows table 5.

Table 5. Intensity of secondary particles at atmospheric depth 200 and $1000 \mathrm{~g} / \mathrm{cm}^{2}$ created by primary particles with energy less than $10.73 \mathrm{GeV}$.

\begin{tabular}{|l|l|l|}
\hline \multirow{2}{*}{ reg. 10} & \multicolumn{2}{|l|}{$I(E<10.73 \mathrm{GeV})$ as rounded \% from $I_{k}(\lambda, x)$} \\
\cline { 2 - 3 } & at $\lambda=200 \mathrm{~g} / \mathrm{cm}^{2}$ & at $\lambda=1000 \mathrm{~g} / \mathrm{cm}^{2}$ \\
\hline Crenkov light & $47 \%$ & $38 \%$ \\
\hline
\end{tabular}




\begin{tabular}{|l|l|l|}
\hline Gamma & $46 \%$ & $6 \%$ \\
\hline electrons & $45 \%$ & $5 \%$ \\
\hline hadrons & $83 \%$ & $56 \%$ \\
\hline muons+ & $46 \%$ & $7 \%$ \\
\hline muons- & $39 \%$ & $2 \%$ \\
\hline positrons & $45 \%$ & $7 \%$ \\
\hline
\end{tabular}

For primary protons in region 10 is $I(E<10.73 \mathrm{GeV})$ equal to $95.9 \%$ of full flux in region 10 . Values in table 4. shows that secondaries from modulated part of primary spectrum create smaller part of secondary flux than in primary flux what leads us to conclusion that variation of secondaries intensity during solar cycle is smaller than variation of primaries intensity.

\section{References}

Aguilar, M., Alcaraz, J., Allaby, J., et al. The alpha magnetic spectrometer (AMS) on the international space station. Part I: Results from the test flight on the space shuttle. Phys. Rep. 366, 331-405, 2002.

Alcaraz, J., Alvisi, D., Alpat, B., et al. Protons in near earth orbit. Phys. Lett. B 472, 215-226, 2000.

Arciprete, F., Bohacova, M., Buonomo, B., et al. AIRFLY: Measurement of the fluorescence yield in atmospheric gases, Proceedings of the 29th ICRC, 7, 55, 2005

Bobik, P., Boella, G., Boschini, M. J., Gervasi, M., Grandi, D., Kudela, K., Pensotti, S., Rancoita, P. G. Magnetospheric transmission function approach to disentangle primary from secondary cosmic ray fluxes in the penumbra region, J. Geophys. Res. 111, A5, CiteID A05205, 2006.

Bobik, P., Boella, G., Boschini, M. J., Gervasi, M., Grandi, D., Kudela, K., Pensotti, S., Rancoita, P. G., Fluxes and nuclear abundances of cosmic rays inside the magnetosphere using a transmission function approach, Advances in Space Research, 43, 3, 385-393, 2009.

Grieder, P.K.F. Cosmic Rays at Earth. Elsevier, Amsterdam, 23, 2001.

Heck, D., Knapp, J., Capdevielle, J. N., Schatz, G., Thouw, T. CORSIKA: a Monte Carlo code to simulate extensive air showers., Forschungszentrum Karlsruhe GmbH, Karlsruhe (Germany), D30167 Hannover, 1998.

IGRF. Web site: http://www.ngdc.noaa.gov/IAGA/vmod/igrf.html, 2010.

International Standard Atmosphere model. Web site: http://www.atmosculator.com/The Standard Atmosphere.html

Masarik, J., Beer, J. Simulation of particle fluxes and cosmogenic nuclide production in the Earth's atmosphere . J. Geophys. Res., 104, D10, 12099-12112, 1999.

Shea, M.A., Smart, D.F., McCracken, K.G. A study of vertical cutoff rigidities using six degree simulations of the geomagnetic field. J. Geophys. Res. 70 (17), 4117-4130, 1965.

Stoermer, K. Periodische Elektronenbahnen im Felde eines Elementar- magneten und ihre Anwendung auf Brches Modellversuche und auf Eschenhagens Elementarwellen des Erdmagnetismus. Mit 32 Abbildungen., Z. Astroph. 1, 237-274, 1930.

Tsyganenko, N.A., Stern, D.P. Modeling the global magnetic field of the large-scale Birkeland current systems. J. Geophys. Res. 101, 27187- 27198, 1996. 
